\title{
22. A Scanning Electron-Microscopic Study on the Endometrium of the Rabbit during the Early Pregnancy
}

\author{
By Tsunenori NaKamura and Hiroyoshi NinomiYA \\ Faculty of Veterinary Medicine, Azabu Veterinary College \\ (Communicated by Sajiro Makino, M. J. A., March 13, 1978)
}

Ovum implantation has long been subjects of special interest in both medical and biological fields in relation to reproduction of mammals in general and animal breeding. The investigations of implantation has been carried out by means of injected preparations (Hafez 1966; Tsutsumi 1966), transmission electron microscopy of ultra-thin sections (Larsen 1974), and by scanning electron microscopy of the endometrial surface (Rubio 1976). The information obtained so far has however remained very limited, particularly on the nature and the change of fine vessels in the endometrium during early pregnancy. To our knowledge, the scanning electron microscopic feature of corrosion casts of fine vessels in the implantation site has not been presented previously in detail in any form. A preliminary study by Mcdonald (1975) on the porcine failed to analyse the nature of delicate change of the fine vascularity. The present study was designed to deal stereoscopically with the nature of the fine vessel in the endometrium of the rabbit during the early pregnancy.

Materials and methods. Twelve pregnant rabbits used for study were at the stage of the 9th-day-post-coitus. They were anesthetized by intravenous sodium pentobarbital $(25 \mathrm{mg} / \mathrm{kg})$ and bled through the common carotid artery. In order to support the analyzation of the fine vascularity established by the injection-replica scanning electron-microscopic method, the left uterine horn was ligated and removed for the light microscopic study. Four micra-paraffine serial sections were stained with hematoxylin-eosin, azan, elastica-Van Gieson and PAS stains. On the other hand, the right uterus was injected with the plastic media through the abdominal aorta at the level anterior to the iliac artery. The plastic material used was methacrylic methyl ester monomer with $1 \%$ benzoyl peroxide as a catalyst, $1 \%$ dimethylaniline as an accelerator, and $15 \%$ dibuthylphthalate as a plasticizer. After the plastic media was polymerized, the specimens were submitted to corrosion with $20 \%$ sodium hydroxide, washed in running water, and then dried. The resin cast specimens were dissected into pieces under a binocular microscope. The piece was mounted on a metal block with an ion sputtering instrument. The 
observation of the specimens thus prepared was made under a scanning electron microscope (JSM-1) with an accelerating voltage of $10 \mathrm{kV}$.

Findings. The uterus vascular system is outlined as follows: The principal source of blood to the uterus is supplied by the
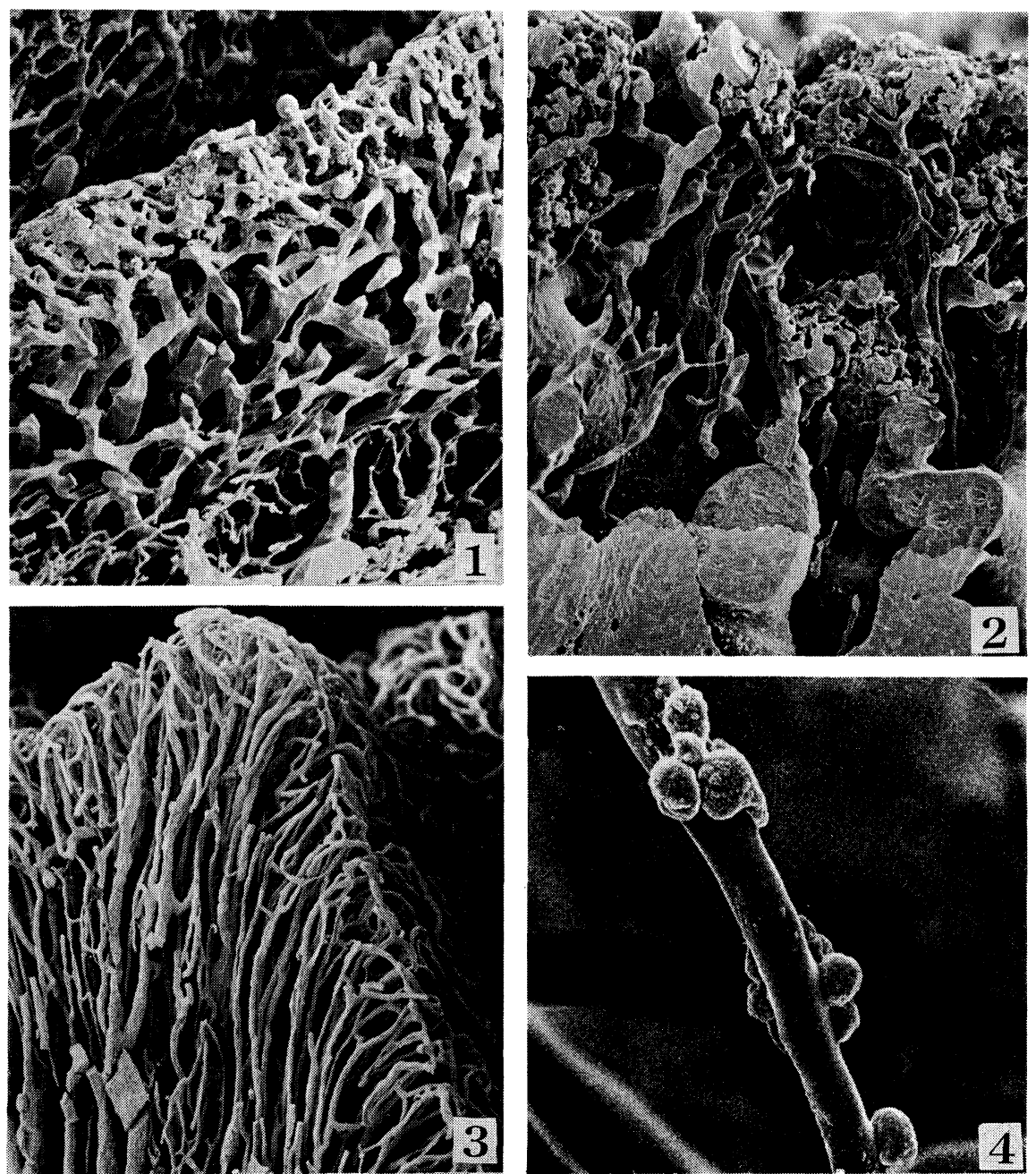

Figs. 1-4. Fig. 1. Vascular system of the endometrium during diestrus. Note the thin subepithelial capillary plexus, in comparison with those in Figs. 2 and 3. (Coronal section, $\times 100$ ). Fig. 2. Vascularity of the placental fold at the 4th-day-post-coitus stage. Note the markedly dilated veins and the blood sinus in the surface capillaries. (Coronal section, $\times 100$ ). Fig. 3. Vasculature of the interconceptus at the 9thday-post-coitus stage. Subepithelial capillary plexus showing an increase of thickness and the well-grown capillaries. (Coronal section, $\times 100$ ). Fig. 4. A high power photomicrograph of the capillary in the periplacental fold, showing bud-like projections. $(\times 1000)$. 
uterine artery formed by a ramification of the urogenital artery which arised from the internal iliac artery. The uterine artery in a slightly waved manner courses cranially in the broad ligament of the uterus to anastomoses with the ovarian artery. In its course the uterine artery branches into many mesometrial arteries, which run around the outer surface of the uterine wall and are continued by the arcuate arteries. These arteries give off many small arteries (radiate arteries) at nearly right angles to the uterine wall and run to the free surface of the epithelium giving off numerous capillaries to the surrounding connective tissue to form an endometrial vascular plexus. The radiate artery also supplies numerous capillaries to the uterine glands forming a subepithelial capillary plexus just under the uterine epithelium. The veins take their origin from these capillary plexuses and run along the corresponding arteries (Fig. 1).

The fine vascularity in the endometrium of the rabbit at the stage of 9-days-post-coitus, 2 days after implantation, was studied. The results of our observations are as follows:

Areas of the conceptus and interconceptus within the uterus were clearly identified. The placental fold in the conceptus was in a well-developed state. The vessels, especially radiate veins, occurring in the fold showed a marked dilation and a slightly convoluted course.

The capillaries in the subepithelial capillary plexus were also welldelineated, showing an elongation into the irregularly arranged plexus. In implantation sites of the placental fold, many small sinusoids, about $100 \mu$ in diameter, were found in the surface capillaries, in a form of transforming into the placental sinuses (Fig. 2).

The obplacental wall was very thin in appearance. The subepithelial capillary plexus and endometrial plexus were failed to define, since they were probably lost completely.

The subepithelial plexus of the interconceptus areas was thick. The capillaries in the plexus were found to be streched and anastomosed with each other to form a complicate capillary network. The radial arteries and veins in this area were well-developed taking a comparatively direct course (Fig. 3 ).

The vascular system in the periplacental fold was similar in structure to that found in the interconceptus areas, while numerous fine bud-like protrusions or surface irregularities were demonstrated in some capillaries near the placental fold. They were about 4 to $10 \mu$ in diameter. Probably they develop into the sinusoids in the capillaries at the implantation stage (Fig. 4).

Remarks. The findings obtained by the present study were in essential feature consistent with those by Hafetz (1966) and Tsutsumi (1966) on the change of the endometrial vascularity during early 
pregnancy in the rabbit, providing in addition some essential findings on the architectural feature of the fine vascular vessels. Numerous fine bud-like protrusions or surface irregularities were observed in the fine blood vessels in the subepithelial plexus of the peri-placental fold. The features here observed may be the first change of the capillaries after implantation. If the artefacts caused by disruption of the vessels through the resin injection were taken into consideration, it would be premature to see that those surface structures represent the fine aneurysm observed under the light microscope, or they are an important structure for the future blood sinus formation or capillary hemorrhages generally found in the earlier stage. Referring to the vascular change, the infiltration of the pseudo-eosinophil cells into the fine vascular wall were observed histologically. Then it is conceivable that these pseudo-eosinophil cells play an important role in aneurysm, the future blood sinus formation and hemorrhages.

Investigations are now in progress in relation to the change of the fine blood vessel in the endometrium of the rabbit during early pregnancy. One of our projects is to analyse the change of the fine vascularity of the endometrium at different stages of pregnancy. Future problems are to deal with the question as to whether the delicate change occurring in the fine vessel of the rabbit is of the similar nature to the evidence found in the rat. Further, our special attention has been paid in relation to the cause of abortion frequently occurring at the end of pregnancy in the rabbit.

In conclusion, it is very probable that the scanning electron microscopy with the resin casts seems to be very useful for the investigation of the nature and course of the change of the fine blood vessel in the endometrium during pregnancy.

Summary. Twelve pregnant rabbits at the stage of the 9th-daypost-coitus were studied on the change of the blood vessel in the endometrium, by means of the scanning electron microscopy with the resin casts. The development of the subepithelial capillary plexus was shown in the placental fold forming a well-defined capillary complex. The surface capillaries comprising the plexus were noted, and some of them showed a change into the small blood sinuses. A number of fine bud-like protrusions or surface irregularities was defined in the capillaries in the region adjacent to the placental fold. Probably they represent minute aneurysm, blood sinus formation or capillary hemorrhage.

Acknowledgement. The authors' cordial thanks are expressed to Emeritus professor Sajiro Makino, M. J. A., the Hokkaido University, for checking the manuscript with invaluable advice. This work was supported by a grant-in-aid (No. 210709) for the Scientific Research from the Ministry of Education, Science and Culture of Japan. 


\section{References}

Hafez, E. S. E. (1966) : Am. J. Anat., 118, 249-282.

Macdonald, A. A. (1976) : Anat. Rec., 184, 689-698.

Larsen, J. F. (1962) : Am. J. Anat., 109, 319-334.

Rubio, C. A. (1977) : Anat. Rec., 185, 359-372.

Tsutsumi, Y. (1966) : J. Morph., 118, 43-56.

_- (1966) : Bull. Cornell Univ., 56, 527-542.

- (1962) : J. Faculty of Agriculture, Hokkaido Univ., 52, 372-482. 